\title{
Casuistique et médiation des savoirs dans la formation professionnelle
}

Knowledge casuistry and mediation in vocational training

Aude Seurrat

\section{OpenEdition}

\section{Journals}

Édition électronique

URL : http://journals.openedition.org/communicationorganisation/5166

DOI : 10.4000/communicationorganisation.5166

ISSN : $1775-3546$

Éditeur

Presses universitaires de Bordeaux

\section{Édition imprimée}

Date de publication : 1 juin 2016

ISBN : 979-10-300-0059-7

ISSN : 1168-5549

\section{Référence électronique}

Aude Seurrat, "Casuistique et médiation des savoirs dans la formation professionnelle »,

Communication et organisation [En ligne], 49 | 2016, mis en ligne le 01 juin 2019, consulté le 04 janvier 2020. URL : http://journals.openedition.org/communicationorganisation/5166 ; DOI : 10.4000/ communicationorganisation.5166 


\title{
Casuistique et médiation des savoirs dans la formation professionnelle
}

\author{
Aude Seurrat ${ }^{-1}$
}

\section{Introduction}

Cet article propose d'exposer et d'illustrer l'usage que nous faisons d'écrits portant sur la casuistique afin d'appréhender la médiation de savoirs prudentiels dans la formation professionnelle. Depuis notre thèse, soutenue en 2009, portant sur des programmes de formation aux médias déployés par l'Union Européenne (Seurrat 2009), nous avons travaillé sur trois autres types de formations professionnelles : la formation via les « serious games " (Labelle, Seurrat 2012), la formation à la communication interculturelle (Seurrat 2012) et la formation à la prise de parole dans les médias². Or, nous avons pu observer, dans tous ces terrains de recherche - et ce malgré leur diversité de formes, d'enjeux et de contextes organisationnels - la volonté d'élaborer des savoirs pratiques et des normes d'action à partir de sélections d'exemples considérés comme typiques. C'est pourquoi, nous proposons dans cet article une réflexion sur les modalités spécifiques de la médiation des savoirs par l'élaboration de cas typiques dans la formation professionnelle. Cet article propose de penser la casuistique comme un processus communicationnel de médiation de savoirs issus de l'expérience concrète et élaborés en cas exemplaires. Notre méthodologie, composite (Le Marec 2002), se basera à la fois sur l'analyse des discours des promesses de dispositifs de formation ${ }^{3}$, sur l'analyse sémiodiscursive d'outils de formation (kits et serious games ${ }^{4}$ ) et sur l'observation

\footnotetext{
1 Aude Seurrat est maître de conférences en sciences de l'information et de la communication et responsable de la Licence «Information et Communication » de l'Université Paris 13, membre du Labsic et du Labex ICCA ; aude.seurrat@univ-paris13.fr

2 Aude SEURRAT, «Circulation et instrumentalisation des savoirs sur la communication dans la formation à la prise de parole dans les médias ", $2^{\mathrm{e}}$ Conférence internationale francophone en sciences de l'information et de la communication, "Intersections, Construction des savoirs en information-communication dans l'espace francophone », Bucarest, 30-31 octobre 2014.

3 Nous nous appuierons pour cela sur une analyse de corpus de sites web et de brochures de présentations de formations à l'interculturel et au mediatraining.

4 Ces exemples seront tirés de notre travail de thèse sur les kits de formation des journalistes développés par l'Union Européenne de Radio-Télévision et sur l'analyse d'une série de serious games de formation menée dans le cadre de notre post-doctorat (projet ManEGE, CNRS, 2010-2012).
} 
participante de plusieurs sessions de formations professionnelles ${ }^{5}$. Notre visée nest pas de présenter de manière exhaustive chaque terrain de recherche un par un, mais de voir, en quoi la casuistique peut servir d'appui heuristique pour appréhender l'élaboration, la catégorisation et l'exemplification de savoirs considérés comme " opérationnels ".

L'objectif de cet article consiste à décomposer les étapes de cette médiation des savoirs en nous appuyant sur ces différents terrains de recherche. Nous nous inscrivons dans la perspective de Jacques Bonnet pour qui « le savoir et les savoirs - professionnels, populaires, artisans, scientifiques, technologiques ou autres - sont approchés en tant que processus " (Bonnet 2015: 64). Les savoirs prudentiels que nous examinons n'existent pas en dehors des contextes, des enjeux, des supports de formation (kits, serious games) et des pratiques de formation (en présentiel ou à distance) qui définissent leurs conditions d'énonçabilité (Foucault 1969: 170). Comme le souligne Yves Jeanneret, "l'expression "médiation des savoirs" suppose que sont produits des savoirs en certains lieux et que ceux-ci sont "médiés", c'est-à-dire qu'un ensemble d'acteurs, d'opérations, de productions contribuent à les faire circuler, à les transformer, les rendant ainsi aptes à une réappropriation $»^{6}$.

Par l'emploi de l'expression "savoirs prudentiels» nous nous référons tout d'abord à la notion de phronesis telle qu'elle est développée par Aristote dans l'Éthique à Nicomaque et telle qu'elle est ensuite analysée par François Jullien dans son Traité de l'efficacité (Jullien 1996). La prudence est une faculté de discernement qui permet la prise de décision. «Est prudent, possède cette capacité pratique, qui est capable de délibérer correctement sur ce qui est bon et avantageux pour lui» (Ethique à Nicomaque, VI,5) $»^{8}$.

D'autre part, par l'emploi de l'expression "savoirs prudentiels », nous nous référons également à la notion de jurisprudence. " En droit romain, l'expression jurisprudentia recouvre l'activité des juriconsultes, des interprètes de la norme ${ }^{9}$. Dans son acception juridique contemporaine, " on dotera la jurisprudence d'une définition procédurale exclusive de toute appréciation ontologique: il s'agit de l'ensemble des processus qui contribuent à garantir l'unification de l'interprétation du droit donnée par les tribunaux. Cette définition conduit à recherche la jurisprudence, non pas dans la somme des décisions rendues par les tribunaux, mais dans celles de ces décisions qui sont

5 Outre les observations participantes dédiées aux formations des journalistes par le Diversity Toolkit (menées dans le cadre de notre thèse), nous nous baserons également sur deux observations participantes récentes de deux formations au mediatraining (formation des militants d'Amnesty International, formation du réseau « les expertes » par le groupe Egalis).

6 Yves JEANNERET, «Le partage des savoirs entre métamorphose des médias et poétique des discours », Médiation et représentation des savoirs, Sous la direction de Jean-Paul METZGER, Paris: L'Harmattan, 2004, p. 21.

7 ARISTOTE, Éthique à Nicomaque, Traduction (1959) de J. Tricot, Éditions Les Échos du Maquis, 2014.

8 Ibid., p. 21.

9 Ferdinand MELIN-SOUCRAMANIEN, « la notion de jurisprudence du conseil constitutionnel », dans Dimitri LAROFF, Manuel RAMIREZ, La pratique constitutionnelle en France et en Espagne, presses universitaires de Bordeaux, 2001, p.199. 
rendues par les juridictions disposant du pouvoir d'imposer une interprétation donnée aux autres tribunaux $»^{10}$. Dans le cadre qui nous occupe, en l'occurrence la formation professionnelle, il ne s'agit pas de formuler un jugement au sens juridique du terme mais d'apprendre à prendre des décisions à partir d'autre cas dont les modes décisionnaires font autorité.

À travers cet article, nous monterons quelles sont les médiations et les enjeux à l'œuvre lors du passage d'un exemple concret à un cas typique et, d'un cas typique à une norme d'action. Nous verrons tout d'abord comment sont sélectionnés des exemples issus de la pratique professionnelle et comment ceux-ci sont construits en cas. Nous nous attacherons ensuite à examiner les processus de décontextualisation et de généralisation relatifs à lélaboration de cas typiques. Enfin, nous questionnerons les prétentions de reproductibilité des actions et l'imposition de normes par la résolution de cas.

\section{La sélection d'exemples considérés comme typiques}

Les formations professionnelles sur lesquelles nous travaillons ont toutes, malgré leur diversité, la prétention de permettre aux formés d'acquérir des savoir-faire et des savoir-être utiles, pour ne pas dire «efficaces », dans l'exercice quotidien de leur profession. Par exemple, le Diversity Toolkit ${ }^{11}$ de l'Union Européenne de Radio-Télévision vise à former les journalistes aux gestes quotidiens qui permettraient d'éviter les stéréotypes dans leur production médiatique. Les serious games de formation promettent, quant à eux, la maîtrise de situations de communication comme l'entretien individuel ${ }^{12}$, le management « de proximité ${ }^{13}$ ou encore la maîtrise des gestes de sécurité à adopter vis-à-vis d'un équipement spécifique ${ }^{14}$. Les formations en présentiel au mediatraining ou à la communication interculturelle promettent, quant à elles, de savoir gérer des situations de communication " typiques » comme l'entretien avec un journaliste ou la négociation avec " un japonais » (Seurrat 2012). Dans tous les cas, la prétention est de donner aux formés

10 Evelyne SERVERIN, "Les divergences de la jurisprudence comme objet de recherche », dans Pascal ANCEL et Marie-Claire RIVIER (dir.), Les divergences de jurisprudence, publications de l'Université de SaintEtienne, collection Droit, 2003, p. 73. (en rouge, cité dans la biblio.).

11 Développé entre 2008 et 2009, ce kit de formation s'adresse à tous les professionnels des chaînes de télévision publiques d'Europe. Par une série d'étude de cas issus des différentes chaînes, il vise à apprendre aux journalistes et, plus largement aux différents professionnels des chaînes de télévision, à intégrer les principes de « lutte contre les discriminations » et de " promotion de la diversité » dans leur pratique quotidienne. Le kit est téléchargeable en ligne sur le site de l'UER http://fra.europa.eu/sites/default/files/fra_uploads/111-mediatoolkit-documentation_en.pdf

12 Nous citerons, par exemple, les portefolios de serious games développés par KTM Advance et constitués de dispositifs de formation numériques sur "l'entretien de management », « l'entretien annuel d'évaluation » ou encore « la client attitude ». La présentation de ces offres peut être consultée sur le site de l'entreprise : http:// www.ktm-advance.com/portfolio/

13 Nous présenterons plus précisément ce serious game dans la suite de l'article. Développé par l'entreprise Unilearning à destination des managers d'une chaîne de supermarchés (dont nous ne pouvons pas citer le nom), ce serious game vise à former au " management de proximité ».

14 Citons par exemple le serious game "Wattou » développé par Sucubus Interactive pour ERDF et qui vise à former les employés d'ERDF au respect des consignes de sécurité lors de la maintenance du réseau électrique. 
des " clés de réussite ", notamment à partir du récit d'exemples considérés comme représentatifs des enjeux de la formation. En cela, ces savoirs peuvent être considérés comme des "savoirs d'action ». Pour Jean-Marie Barbier, " traditionnellement assimilés aux compétences pratiques, aux savoirs cachés, aux savoirs d'expérience, aux savoirs informels, aux habilités acquises dans l'action, les savoirs d'action ou les savoirs relatifs aux transformations du réel donnent lieu (...) à énoncé et à formalisation, ce qui tend à les affermir dans leur statut de savoirs et à les rapprocher des savoirs théoriques " (Barbier 1996). En cela, la démarche casuiste que nous analysons consiste justement à formaliser des situations issues de la pratique afin de les ériger en savoirs d'action.

La volonté d'élaborer des solutions concrètes d'application des principes dans la pratique, de proposer de résoudre des cas présentés comme exemplaires peut être appréhendée comme une forme contemporaine de casuistique. La casuistique est l'art d'appliquer les lois générales d'une discipline à un fait. À la fin du XVI ${ }^{\mathrm{e}}$ siècle, en Occident, elle était la méthode presque exclusive de la théologie morale catholique. Des cours de «cas de conscience» étaient inscrits aux programmes des séminaires et s'appuyaient sur des manuels d'études de $\operatorname{cas}^{15}$. Ces manuels de casuistique avaient vocation à servir de guides d'action. Les casuistiques morales et religieuses, telles que les ont approchées notamment les écrits de Jean-Claude Passeron et de Jacques Revel (Passeron, Revel 2005), de Pierre Cariou (Cariou 1979) et de Pierre Hurtubise (Hurtubise 1993), peuvent être mobilisés comme appui heuristique pour penser la circulation du normatif à l'empirique, l'apprentissage, par l'étude de cas, de gestes du quotidien destinés à concrétiser des normes. Il s'agit ainsi d'aménager les voies d'application du principe, "rendre la règle applicable malgré les contraintes de l'occasion » (Cariou 1993: 45). Les cas traités servent d'exemples qu'il convient d'imiter dans des cas similaires. "La méthode casuistique consiste à résoudre les problèmes posés par l'action concrète au moyen de principes généraux et de l'étude des cas similaires. Deux principes la fondent : validité des lois générales comme normes de l'action particulière ; similitude de certaines actions humaines qui permet de transposer les lois de l'agir de l'une à l'autre ${ }^{16}$. Nous passons alors du particulier au général, le cas devenant un principe d'action généralisable. Ainsi, cette "étoprudence » (Hurtubise 1993 : 243) ou « jurisprudence spirituelle ( Hurtubise: 278) a pour objectif de guider la pratique du confesseur à partir de résolutions de cas considérés comme similaires et érigés en modèles d'action.

Deux formes de casuistique que nous retrouvons dans nos dispositifs de formation sont à distinguer : la casuistique typique et la casuistique de "cas de figure ». Comme le souligne Serge Boarini, "le cas est ce qui "tombe"

15 Le plus connu étant le Dictionnaire des cas de conscience paru à Paris en 1717 (réédité en 2012 : Jean Pontas , Eusebius Amort, Abbé Vermot, Dictionnaire des cas de conscience, Nabu Press, 2012).

16 Encyclopaedia Universalis, vol. 3, 1968, p. 1105. 
[cadere], ce qui tombe à pic soit parce qu'il tombe à côté de la norme de conduite prescrite: le cas serait alors l'exception surprenante et irréductible faite à la norme, la marque de l'irrégularité de la norme; soit parce qu'il tombe bien de sorte que tous conviennent de ce qu'il faut faire: le cas serait alors exemplaire de la norme $»^{17}$. Les formations étudiées mobilisent surtout des " configurations typiques. La configuration typique correspond aux situations habituelles de la pratique : le cas est présenté comme étant le représentant des situations courantes. Les « cas de figure " sont liés, quant à eux, aux situations exceptionnelles souvent qualifiées « de crise ». Ceci correspond la plupart du temps au découpage des formations en deux niveaux que nous rencontrons notamment dans les offres de formation au mediatraining. Les formations de niveau 1 proposent de maitriser les situations « normales » de prise de parole dans les médias et les formations de niveau 2 proposent de s'exercer sur des mises en situations plus complexes ou « de crise ». Dans les deux situations, l'exemple est institué en cas pour permettre de dégager des modes de résolution. Dans les configurations typiques, « entendu comme exemplaire, le cas devient l'occasion de l'application de la norme. Dans le cas exemplaire, toute la norme se réalise de sorte qu'il devient le modèle de toute réalisation à venir. À ce moment de la casuistique correspond la recherche de cas paradigmatiques. Ces cas dépassent la singularité des cas collectionnés par les dictionnaires ou les recueils pour qu'on en retire ce qui pourrait être appliqué aux occurrences semblables futures ${ }^{18}$. Il s'opère alors une dialectique entre le normatif et l'empirique puisque l'empirique est évalué à l'aune du normatif puis érigé en exemplum, comme modèle à promouvoir. Dans " l'Ancienne Rhétorique ", Roland Barthes se penche sur la figure de l'exemplum et explique qu'il s'agit de "l'induction rhétorique : on procède d'un particulier à un autre particulier par le chaînon implicite du général : d'un objet on infère la classe, puis de cette classe on défère un nouvel objet. [l'exemplum] est une similitude persuasive, un argument par analogie : on trouve de bons exempla, si on a le don de voir des analogies - et aussi, bien entendu, les contraires- comme son nom l'indique, il est du côté du paradigmatique, du métaphorique » (Barthes 1970: 200).

La première étape de la démarche casuiste consiste à repérer des exemples issus de la pratique professionnelle et qui correspondent aux objectifs et questions posés par la formation. Le repérage de ces exemples potentiels s'effectue soit grâce à une collecte d'exemples venants de différents terrains, soit grâce à des enquêtes menées sur le terrain du commanditaire de la formation, soit à partir de l'expérience des formateurs eux-mêmes. Nous trouvons ces trois types de recherches d'exemples à typifier dans nos terrains de recherche. Le Diversity Toolkit de l'UER a été élaboré à partir d'une collecte et d'une

17 Serge BOARINI, «Collection, comparaison, concertation. Le traitement du cas, de la casuistique moderne aux conférences de consensus », Jean-Claude PASSERON, Jacques REVEL, op. cit., p. 130.

18 Ibid., p. 139. 
sélection de programmes télévisés issus de différentes chaînes publiques d'Europe. Chaque chaîne était invitée à envoyer des programmes portant sur les différences culturelles qui soit avaient fait l'objet de plaintes soit étaient considérées par les chaînes elles-mêmes comme des "bonnes pratiques ". Quant aux entreprises qui proposent des serious games de formation, elles élaborent ceux-ci, la plupart du temps, à partir d'enquêtes de terrains menées dans l'entreprise du commanditaire de la formation. Ces enquêtes ont à la fois pour objectif de faire ressortir les normes qui régissent la profession ou le secteur d'activité en question et d'extraire des situations et des pratiques professionnelles types qui les mettent en jeu. Par exemple, le serious game destiné aux managers de supermarchés est construit autour d'une " journée type » d'un manager et met à l'épreuve le joueur-manager sur un certain nombre de situations-types de management (recadrer un employé en retard, rappeler les règles d'hygiène et de sécurité aux équipes, etc.). Enfin, dans les formations aux relations interculturelles (souvent destinées à de futurs expatriés) ou dans les formations au médiatraining, les formateurs sélectionnent des exemples de leur propre pratique professionnelle (de leur expérience à l'étranger pour les formateurs à l'interculturel ou de leur expérience journalistique pour les formateurs en mediatraining) afin de les ériger en cas typiques de problèmes rencontrés dans ce domaine d'activité concerné.

\section{Les processus de décontextualisation et d'exemplification}

Une fois l'exemple sélectionné, celui-ci n'a pas en lui-même une valeur d'exemplarité. Ce sont donc les opérations de réécritures qui sont autant de médiations de l'exemple au cas typique qui permettent de lui attribuer une certaine exemplarité. Pour Serge Boarini, «le cas est d'abord le récit du cas ${ }^{19}$. En effet, le cas ne se réduit pas à un fait, il est constitué de tous les discours qui participent à le raconter, le problématiser, le généraliser et le résoudre. La question est alors celle des modalités de généralisation du cas. C'est justement cette question qui est au cœur de louvrage collectif Penser par cas. Comment une singularité peut-elle permettre un raisonnement plus général? JeanClaude Passeron et Jacques Revel expliquent que

sous des formes qui ont été fort diverses tout au long de l'histoire des savoirs et des savoir-faire, la "pensée par cas" révèle les particularités logiques propres à tout raisonnement qui, placé devant une singularité observable, choisit d'approfondir ses propriétés particulières pour fonder sur leur description, leur interprétation ou leur évaluation, une argumentation de portée plus générale (Passeron, Revel 2005 : 285).

19 Serge BOARINI, «Collection, comparaison, concertation. Le traitement du cas, de la casuistique moderne aux conférences de consensus », dans Penser par cas, op.cit., p.139. 
Une première opération de réécriture du cas consiste à le décontextualiser pour rendre le cas plus général. Cette décontextulisation consiste à " gommer» les éléments qui sont trop spécifiques au cas et qui ne s'appliquent pas à d'autres cas considérés comme similaires. Le cas gagne en généralité car il est réécrit en dehors des contextes spécifiques, des situations organisationnelles, des personnalités des acteurs en présence, etc. Par exemple, dans le Diversity Toolkit, les extraits médiatiques sont sortis de leurs contextes nationaux et éditoriaux de diffusion et sont redocumentarisés en une série documentaire organisée en fiches thématiques. L'enjeu est ainsi de transformer des situations précises en des situations types. Les situations sont exemplifiées car la réécriture ne retient que l'idée et non les spécificités contextuelles de sa mise en place. Cette réécriture semble essentielle car elle permet de situer les savoirs extraits du cas réel dans un contexte d'apprentissage. Comme le souligne Yves Jeanneret, «la réécriture (est) au cœur des disciplines de la trivialité. En effet, la réécriture remplit plusieurs fonctions à la fois: elle socialise une interprétation et une lecture, permet au texte de migrer d'un contexte social à l'autre, d'un milieu à l'autre et rend le texte maniable en le miniaturisant, ce qui est indispensable à son intégration dans des entités culturelles nouvelles $»^{20}$ (Jeanneret $2008: 72$ ).

Prenons ici l'exemple du serious game de formation des managers d'un supermarché au «management de proximité». L'univers visuel est une simulation d'un magasin. Le jeu s'adresse à un public qui connaît déjà l'entreprise et ses règles de fonctionnement. Le joueur joue son propre rôle - en l'occurrence celui d'un manager de magasin- et le scénario mine une journée-type de cette fonction: de l'arrivée dans les bureaux, à la gestion des problèmes au sein du magasin en passant par le brief des employés. Les situations à résoudre sont par exemple, le recadrage d'une employée qui arrive souvent en retard ou la discussion avec une cliente insatisfaite, la modération du conflit entre deux employés. Dans ces cas, des variables contextuelles comme la personnalité des individus, les précédents dans leur relation ne sont pas pris en compte, la situation étant présentée comme une situation-type. Le dispositif vidéoludique vise à former ses usagers à une meilleure mise en pratique des normes professionnelles (propreté, sécurité, etc.), à une meilleure gestion de ses équipes (adopter un «management de proximité») et, par là même à une meilleure adéquation avec la culture d'entreprise.

Ainsi, l'exemple devient cas grâce à toute une série de médiations qui visent à le décontextualiser, le généraliser afin de lui donner sa valeur d'exemplarité. Or, ces opérations sont à la fois des opérations narratives (savoir sélectionner et raconter les éléments pour les reconfigurer selon l'objectif escompté21 [Ricœur 1991]) et rhétoriques (savoir argumenter sur l'intérêt du cas choisi). Elles sont

20 Yves JEANNERET, Penser la trivialité: la vie triviale des êtres culturels, Paris: Hermès, Lavoisier, Coll. Communication, médiation et construits sociaux., p. 72.

21 Il s'agit du deuxième temps de la Mimesis tel qu'il est décrit par Paul RICEEUR. Le philosophe explique que la mise en récit permet « une synthèse de l'hétérogène », le passage d'une « succession » à une " configuration ». 
aussi, dans le cas des kits de formation et des serious gamme des opérations documentaires qui rééditorialisent les pratiques dans l'espace d'écriture des kits ou dans l'univers vidéoludique des serious games.

\section{Idéal de reproductibilité et construction de normes professionnelles}

Qu'ils soient racontés ou mis en pratique par des exercices de « simulation » virtuels ou réels, les cas ainsi élaborés ont pour fonction de servir de modèles dans des situations professionnelles qui auraient des caractéristiques similaires. La compréhension d'un cas doit permettre l'intelligibilité d'un autre. L'enjeu est donc celui de la reproductibilité des pratiques et par là même des normes qui leur président.

Cet idéal de reproductibilité des pratiques vient en quelque sorte, conjurer les angoisses relatives à l'incertitude de l'univers professionnel. Le cas est autant un récit qu'une prescription. Pour Jacques Bonnet, " par prescription, nous entendons les commandes plus ou moins formelles d'activité, d'intervention ou de comportement, commandes dont l'origine peut être hiérarchique du fait de décideurs, ou bien réglementaire ou encore se situer dans le registre d'un changement de politique ou de technicité ou de tout autre événement appelant une réponse en termes de production de sens par des acteurs invités ou contraints à agir » (Bonnet 2005: 68).

Les différents cas traités dans le Diversity Toolkit aboutissent à l'élaboration d'une "check list de la diversité», qui reprend toute une série de questions que doit se poser le journaliste afin d'éviter les stéréotypes et «promouvoir la diversité» dans ses productions médiatiques. Quant aux serious game de formation, ils font, dans la grande majorité des cas, un retour au joueur après chaque action menée afin de dire si celle-ci est ou non en adéquation avec les normes de la profession ou de l'activité (comme par exemple dans le serious game pour les managers de supermarchés, l'usage assez récurrent de la phrase "vous n'avez pas été assez empathique avec votre salarié »). Dans les «mises en situation " proposées dans les formations à l'interculturel ou au mediatraining, le formateur conclut sur « la bonne pratique " à adopter afin d'optimiser sa communication (comme savoir ne pas répondre à une question gênante d'un journaliste ou savoir mettre son interlocuteur étranger « en confiance»).

Les séries de cas viennent ainsi argumenter sur l'importance de la norme et son efficacité communicationnelle. Mais, l'exemplarité n'est pas qu'une illustration de la norme, il s'agit d'une construction complexe qui est également un réinvestissement de la norme.

Lénoncé descriptif normatif est pris dans sa fonction perlocutoire: l'énoncé du cas typique construit le cas, comme il construit tout cas semblable qui pourrait se produire à l'avenir. (...) Dès lors, le cas ne peut plus être illustration a priori de la norme. Il est nécessairement une reformulation de la norme par l'interprétation quen donneront les membres placés dans une situation morale difficile ${ }^{22}$.

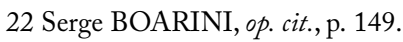


Plus que de donner une série de points de vigilance au journaliste, le Diversity Toolkit construit en creux le portait d'un journaliste modèle qui saurait faire fi de tous ses préjugés, traiter les informations en toute «objectivité» et, dans le même mouvement, promouvoir le «vivre-ensemble» dans ses productions. Quant au serious game pour les managers de supermarchés, il propose en quelque sorte une injonction paradoxale: le manager doit être très réactif, presque doté d'ubiquité et en même temps prendre le temps de développer des relations de proximité avec ses employés et ses clients. Enfin, dans les formations à l'interculturel et au mediatraining, les cas abordés proposent également en creux le portait du professionnel performant, sachant s'adapter aux situations nouvelles et surtout sachant aboutir à ses fins (par exemple conclure efficacement une négociation avec un étranger ou encore faire passer «son message» lors d'une interview avec un journaliste).

La médiation des savoirs par la casuistique a ainsi pour objectif de faire acquérir aux formés des savoir-faire ou, pourrait-on dire des «savoir-résoudre » dans des situations considérées comme typiques, mais elle concerne aussi le devoir-être: les savoir-faire sont au service des devoir-être.

\section{Conclusion: casuistique et normalisation des pratiques socio- professionnelles}

À travers cette démonstration, nous avons pu appréhender la casuistique comme processus de médiation de savoirs prudentiels qui vient normaliser les pratiques socioprofessionnelles. Par leurs dimensions narratives, ces casuistiques professionnelles sont également des formes d'écriture des situations de communication. Écriture documentaire de situations de communication journalistiques pour le Diversity Toolkit, écriture vidéoludique de situations qui définissent un certain ethos professionnel dans les serious game, ou encore mises en scène de «simulations» de pratiques de communication dans les formations en présentiel. Or, ceci révèle une certaine conception des pratiques de communication comme pouvant être modélisées en séries de situations-types.

Cet article n'avait pas pour objet d'appréhender toutes les formes de médiation des savoirs à l'œuvre dans la formation professionnelle. La médiation par la casuistique est une modalité spécifique qui s'articule avec d'autres modalités de médiations comme celle de la médiation de savoirs « savants » (Darbellay 2012), issus notamment des travaux académiques. Ces types de savoirs ne sont pas toujours mobilisés dans les formations étudiées car celles-ci insistent surtout sur leur visée " pratique ». Mais, dans les formations au mediatraining et de manière encore plus marquée dans les formations à l'interculturel, le savoir " savant » vient souvent cautionner en amont, en quelque sorte, les savoirs prudentiels. Il serait ainsi intéressant d'explorer les modes d'articulation entre ces formes de médiations distinctes que sont la casuistique et la vulgarisation voire la modélisation des savoirs académiques au sein de la formation professionnelle. 


\section{BIBLIOGRAPHIE}

ANCEL Pascal et RIVIER Marie-Claire (dir.), Les divergences de jurisprudence, publications de l'Université de Saint-Étienne, collection Droit, 2003.

BARBIER Jean-Marie (dir.), Savoirs théoriques et savoirs d'action, PUF, collection Education et formation, 1996.

BARTHES Roland, «L'Ancienne rhétorique », Communications n 16, 1970.

BONNET Jacques, «L'intelligence du social dans les organisations », Communication E Organisation, n 47, 2015.

CARIOU Pierre, Les idéalités casuistiques, Paris, Éditions Champion, 1979.

CARIOU Pierre, Pascal et la casuistique, PUF, Questions, Paris, 1993.

DARBELLAY Frédéric (dir.), La circulation des savoirs. Interdisciplinarité, concepts nomades, analogies, métaphores, Berne, Peter Lang, 2012.

FOUCAULT Michel, L'archéologie du savoir, éditions Gallimard, Paris, 1969.

HURTUBISE Pierre, La casuistique dans tous ses états, Paris, Novalis, 1993.

JEANNERET Yves, Penser la trivialité : la vie triviale des êtres culturels, Paris: Hermès, Lavoisier, Coll. Communication, médiation et construits sociaux, 2008.

JULLIEN François, Traité de l'efficacité, Livre de poche, Grasset, 1996.

LE MAREC Joelle, "Ce que le terrain fait aux concepts: vers une théorie des composites », Habilitation à diriger des recherches, université Paris 7, 2002.

LABELLE Sarah et SEURRAT Aude, «Médiations ludiques et activités d'apprentissage: réflexions à partir d'une expérience de conception d'un serious game», RIHM, décembre 2012. Vol 13 http://europia.org/RIHM/

PASSERON Jean-Claude, REVEL Jacques, Penser par cas, Paris, Éditions de l'École des hautes études en sciences sociales, 2005.

PONTAS Jean, AMORT Eusebius, VERMOT (abbé), Dictionnaire des cas de conscience, Nabu Press, 2012.

RICCEUR Paul, Temps et récit II, La configuration dans le récit de fiction, Paris, Seuil, 1991

SEURRAT Aude, « Formations à l'interculturel et conceptions de la communication », Revue Économique et Sociale, volume 70, septembre 2012.

SEURRAT Aude, "Les médias en kits pour promouvoir la diversité». Étude de programmes européens de formation aux médias destinés à «lutter contre les discriminations» et à "promouvoir la diversité». Thèse de doctorat, Paris IV, CELSA, décembre 2009. Thèse publiée en ligne sur e-Sorbonne : http://www.e-sorbonne.fr/sites/ www.e-sorbonne.fr/files/theses/THESE-SEURRAT.pdf

SEURRAT Aude, "Les médias en kits pour promouvoir la diversité», Les enjeux de l'information et de la communication, novembre 2010, http://w3.u-grenoble3.fr/les_ enjeux/2010/Seurrat/home.html 
Résumé: Cet article se penche sur la médiation de savoirs prudentiels dans la formation professionnelle. En prenant appui sur des travaux portant sur la casuistique, il questionne l'élaboration de savoirs pratiques et de normes d'action à partir de sélections d'exemples considérés comme typiques. La casuistique est envisagée comme un processus communicationnel de médiation de savoirs issus de lexpérience concrète et élaborés en cas exemplaires. Synthèse de plusieurs terrains de recherche sur la formation des journalistes à la lutte contre les discriminations, la formation via les serious games, la formation au media training et la formation à l'interculturel, cet article propose de décomposer les étapes de cette forme spécifique de médiation des savoirs. Nous verrons tout d'abord comment sont sélectionnés des exemples issus de la pratique professionnelle et comment ceux-ci sont construits en cas typiques. Nous nous attacherons ensuite à examiner les processus de décontextualisation et de généralisation relatifs à lélaboration de cas typiques. Enfin, nous questionnerons les prétentions de reproductibilité des actions et l'imposition de normes par la résolution de cas.

Mots-clés : Casuistique, médiation, formation, exemplarité, norme.

Summary: This article focuses on how practical knowledge is transmitted in professional training. Building on the work on casuistry, it questions the development of practical knowledge and action standards based on examples considered as typical. Casuistry is seen as a communication process of transmission of knowledge coming from practical experience developed as exemplary cases. This article crosses several research fields about journalists' training -fighting discrimination, serious games, media training and sensitization to the intercultural- to analyze the different steps in this particular form of knowledge transmission. We will first see how examples are selected from professional practice and how they are developed as typical cases. We will then move on to an analysis of the process of decontextualisation and generalization in the development of typical cases. Finally, we will question the claims of reproducibility of actions and the imposition of standards by the resolution of cases.

Keywords: casuistry, knowledge transmission, training, exemplary, standard. 